\title{
APPENDIX 1: VERSIFICATION
}

Versification, the Mosul text compared with 7a1 (Leiden edition).

Leiden

1 Chronicles

$1: 51$

$3: 7$

$3: 8$

$3: 23$

$3: 24$

$4: 15$

$4: 24$

$4: 25$

$4: 26$

$4: 32$

$5: 15$

$5: 19$

$5: 27-6: 14$

$6: 15$

6:16-20

$6: 21$

6:22-30

$6: 31$

$6: 32-42$

$6: 43$

$6: 44-50$

6:51

6:52-66

$7: 4$

$7: 5$

$7: 17$

$7: 25$

$7: 26$

$7: 39-40$

$8: 7$

8:9

$8: 23$

$8: 24$

\section{Begins with Mosul}

last 2 words of 1:50

2nd word of $3: 7$

last word of $3: 6$

last 2 words of $3: 22$

$3: 23$

5th word of $4: 15$

5 th word of $4: 21$

4:26

7 th word of $4: 26$

last word of $4: 31$

last 6 words of $5: 14$

5 th word of 5:19

6:1-29

5 th word of $6: 30$

6:31-35

3rd word of $6: 36$

$6: 37-45$

last 2 words of $6: 45$

6:47-57

3 rd word of $6: 58$

6:59-65

last 3 words of 6:65

6:67-81

4th word of $7: 4$

last word of $7: 4$

4 th word of $7: 17$

last 2 words of 7:24

2nd word of $7: 26$

7:34-35

last word of $8: 6$

8:7

3rd word of $8: 16$

2nd word of 8:24 
$8: 26$

$8: 32$

$9: 28$

$9: 31$

9:38

11:2

11:44

11:46

12:5

12:6-20

12:21

12:22-41

$13: 3$

15:5

5:19

15:20

15:21

16:17

16:22

23:5

24:21

24:22

24:25

$25: 1$ (p. 61) +24:26.30-31+25:1

(p. 62)

26:16

26:17

26:20

26:26

26:27

27:21

28:11-19

29:19

29:21

\section{Chronicles}

1:18

2:1-17

$4: 18$

10:3

$1 \operatorname{Rg} 12: 21-30$ (pp. 97-98)

$1 \operatorname{Rg} 13: 34$ (p. 98)

$1 \operatorname{Rg} 14: 1-9$ (p. 98)

13:12

13:23-14:10

14:11 last word of 8:25

last word of $8: 31$

4th word of 9:28

7 th word of $9: 30$

2nd word of 9:38

last 3 words of 11:1

2nd word of 11:44

2nd word of 11:46

last 4 words of 12:4

12:5-19

8 last words of 12:19

12:21-40

4th word of 13:3

last 2 words of 15:4

6th word of 15:19

8th word of 15:20

3 rd word of 15:21

last 3 words of 16:16

last 2 words of 16:21

not marked in $\mathrm{L}$

last word of 24:20

5 th word of $24: 21$

last word of 24:24

25:1

4th word of $26: 16$

4th word of 26:17

last word of 26:19

3 rd word of $26: 26$

3rd word of 26:27

7 th word of $27: 21$

28:11-12 + 15-17

6th word of 29:19

last 4 words of 29:20

2:1

2:2-18

4:12

5th word of 10:2

11:1-10

11:10

11:11-17

last 7 words of 13:12

14:1-11

7 th word of $14: 12$ 
14:12-14

15:7

15:19

19:7

19:9

20:17

$20: 26$

22:4

22:5

$23: 2$

24:25

24:27

25:21

26:14

26:18

$27: 6$

$27: 9$

28:15

28:22

28:23

28:25

29:33

30:11

$30: 12$

$31: 4$

$31: 8$

$31: 17$

$31: 19$

32:24

$32: 25$

$33: 17$

$34: 4$

$35: 12$

$35: 15$
14:13-15

last 5 words of $15: 7$

16:1

9th word of 19:7

3rd word of 19:9

last 4 words of 20:16

13th word of 20:26

5 th word of 22:4

last 4 words of 22:4

5th word of 23:2

last 5 words of $24: 24$

last 5 words of $24: 26$

4 th word of 25:20

not marked in $\mathrm{L}$

last 2 words of $26: 17$

4th word of $27: 6$

$27: 8$

$28: 14$

28:15

4th word of $28: 23$

8th word of $28: 25$

3rd word of 29:33

6th word of 30:11

8th word of $30: 12$

7 th word of $31: 4$

last 5 words of $31: 7$

7 th word of $31: 17$

4th word of 31:19

4 th word of $32: 24$

last 6 words of 32:24

last 2 words of 33:16

3rd word of $34: 4$

3rd word of 35:12

last word of 35:14 
